Research Paper

\title{
Surgical Treatment and Outcomes of Calcaneal Osteomyelitis in Adults: A Systematic Review
}

\author{
Marta Sabater-Martos ${ }^{1,2}$, Irene Katharina Sigmund ${ }^{1,3}$, Constantinos Loizou ${ }^{1}$, Martin McNally1 ${ }^{1 凶}$ \\ 1. The Bone Infection Unit, Nuffield Orthopaedic Centre. Windmill Rd, Headington. Oxford OX3 7HE, Oxford University Hospitals Foundation NHS Trust, \\ Oxford, UK. \\ 2. Department of Orthopaedics and Trauma Surgery. Hospital Universitari Germans Trias i Pujol, Badalona. Carretera del Canyet s/n, 08916 Badalona, Spain. \\ 3. Medical University of Vienna, Department of Orthopaedics and Trauma Surgery, Spitalgasse 23, 1090 Vienna, Austria. \\ $\square$ Corresponding author: Mr. M.A. McNally; email: martin.mcnally@ouh.nhs.uk
}

(c) The authors. This is an open access article distributed under the terms of the Creative Commons Attribution (CC BY) license (https://creativecommons.org/licenses/ by/4.0/). See http://ivyspring.com/terms for full terms and conditions.

Received: 2019.02.27; Accepted: 2019.03.23; Published: 2019.05.21

\begin{abstract}
Introduction: Calcaneal osteomyelitis is an uncommon and challenging condition. In this systematic review we aim to analyse the outcomes from concomitant use of bone debridement and soft tissue management for patients diagnosed with calcaneal osteomyelitis.

Materials \& Methods: A complete computerised and comprehensive literature search of Pubmed and Cochrane database was undertaken from January 2000 to October 2018. During the review, studies were screened for information about the surgical and antimicrobial treatment, the complications, the reinfection rate and the functional outcome of patients with calcaneal osteomyelitis.

Results: Of the 20 eligible studies included, seven (35\%) described bone treatment only, six (30\%) soft tissue treatment only, five (25\%) soft tissue and bone treatment, and two (10\%) focused on prognostic factors and differences in outcomes between diabetic and non-diabetic patients.

In the studies with bone treatment only, infection recurrence ranged from 0 to $35 \%$ and the amputation rate from 0 to $29 \%$. If soft tissue coverage was also needed, both the reinfection rate and amputation rate ranged from 0 to $24 \%$. Studies presenting the functional status generally showed preservation or even improvement of the preoperative ambulatory status.

Conclusion: Calcaneal osteomyelitis is difficult to treat. A multidisciplinary approach involving orthopaedic surgeons, plastic surgeons and infectious disease physicians is preferred. The heterogenicity of studies has hindered the development of agreed treatment protocols, which would be useful in clinical practice.
\end{abstract}

Key words: calcaneal osteomyelitis, systematic review, outcome, treatment, muscle flap, antimicrobial carrier, infection.

\section{Introduction}

Calcaneal osteomyelitis poses specific challenges for patients and treating clinicians. Of all osteomyelitis cases, the incidence ranges from 3 to $10 \%$ (1) and is often seen in association with diabetes and other comorbidities. Infection most commonly presents after a traumatic event (open fractures or following fixation) or in patients with heel ulceration due to prolonged bed rest or lower limb neuropathy or vasculopathy (1). The diagnosis is primarily based on clinical features (unilateral localized swelling, erythema, localized pain, increased limb temperature, sinus or heel ulceration). Radiological assessment with CT or MRI (Figure 1) and bacteriological analysis can help to confirm the diagnosis (2). The calcaneum is unusual, in that, it has specialised skin attached very tightly to the bone surface with short, dense fibres. Infection within the bone rarely causes sub-periosteal abscesses, but rather erodes through the specialised skin producing ulceration and skin loss (3). It is very difficult to restore this tissue loss (Figure 2). For successful treatment of calcaneal osteomyelitis (infection control and good functional 
outcome) the following objectives should be delivered:

- excision of all necrotic bone and soft tissue

- good dead space management

- preservation of weight bearing capability

- good soft tissue cover for a well vascularised environment (2).

Nevertheless, treatment options for calcaneal osteomyelitis are limited. Historically, the standard treatment has been lower limb amputation. This treatment is effective in infection eradication but is associated with an inefficient gait (4) as well as reduced physical activity (5) and mortality rates approaching $70 \%$ at 5 years in diabetic patients (6). In addition, in patients who cannot apply or remove a prosthesis, can result in them being bed bound or wheelchair bound.
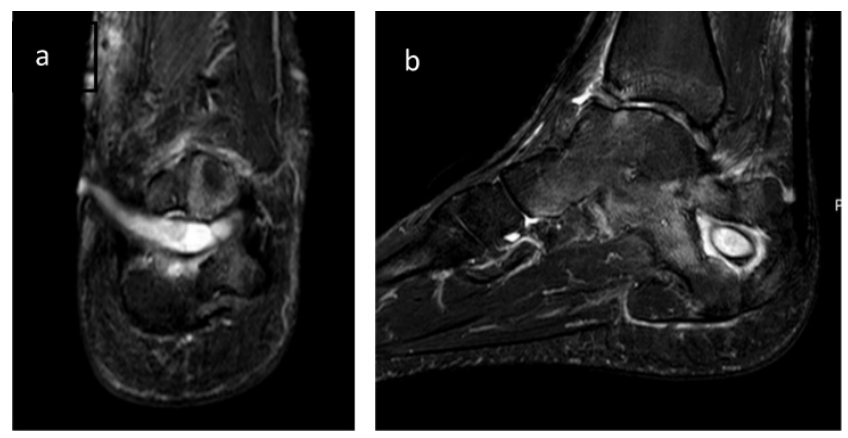

Figure 1. 1a: Coronal MRI showing central abscess with sinus extending to the medial side. There is no subperiosteal extension of the abscess. This infection followed internal fixation of a closed, complex calcaneal fracture. 1b: Sagittal MRI showing the classical 'corona' sign of osteomyelitis.
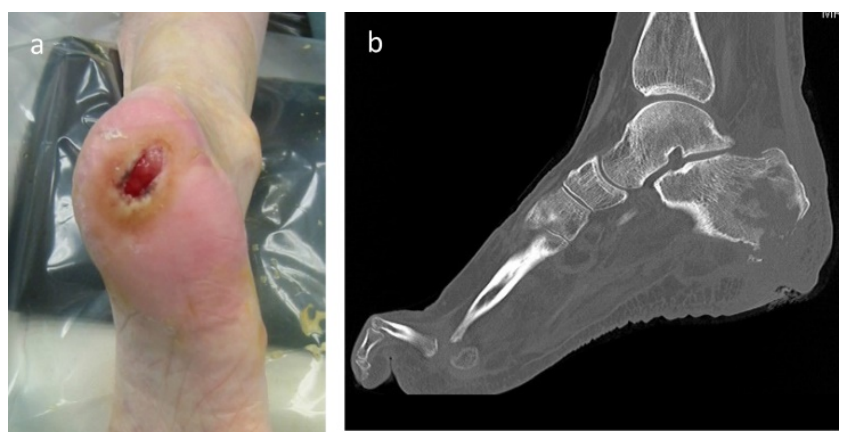

Figure 2. 2a: Plantar ulceration over calcaneal osteomyelitis in a patient with paraplegia after spinal cord injury. 2b: CT Scan showing the bone destruction deep to the ulcer.

Therefore, appropriate limb salvage procedures for treatment of calcaneal osteomyelitis are of immense importance to achieve infection-free conditions which offer a good functional outcome for these patients. Treatments may involve not only bone debridement techniques such as curettage, partial or total calcanectomy, but also soft tissue management.
A great range of treatments are described from primary wound closure or delayed primary wound closure to negative pressure therapies (NPWT), pedicle flaps and microsurgical free flaps $(7,8)$. Nevertheless, evidence on the efficacy and accuracy of combining both treatments are lacking.

The aim of this review was to analyse the concomitant use of bone and soft tissue approaches for patients diagnosed with calcaneal osteomyelitis.

\section{Materials and method}

\section{Search strategy}

A computerised literature search in Pubmed and the Cochrane library database was conducted. We included articles published in English during the last 19 years, from January $1^{\text {st }} 2000$ to October 31 2018. Search terms included "calcaneum", "calcaneal" "osteomyelitis", "osteitis". Calcaneal osteomyelitis in children often has a different aetiology, treatment and prognosis, compared to adult calcaneal osteomyelitis. For this reason, this review does not include cases of calcaneal osteomyelitis during childhood (patients under 16 years old). A full search strategy is shown in figure 3 . Any animal or in vitro studies were excluded. All levels of evidence except case reports were included. Articles not providing a clear description of the surgical treatment and/or outcome were excluded. Finally, studies not providing an abstract were also excluded. Further studies identified from reference lists were added after review of full papers.

\section{Study selection}

A total of 227 studies were identified from our search. Of these, 162 publications did not meet the inclusion criteria and were eliminated. Full text articles were retrieved for 23 publications. Of these, four were excluded because two did not provide a clear description of the surgical treatment and two were overviews of the condition. Thus, 19 studies met the inclusion criteria and were reviewed in detail. References from these studies were analysed and one further study was included in the review.

\section{Data extraction}

The following data was extracted from all relevant papers: 1) author and journal, 2) year of publication, 3) type of study, 4) number of patients, 5) treatment received, including information on bone resection and debridement, soft tissue and dead space management, antimicrobial therapy and use of adjunctive therapy such as negative pressure wound therapy, growth factors or external fixators, 6) complications, 7) outcome, 8) recurrence of infection and 9) study limitations. 


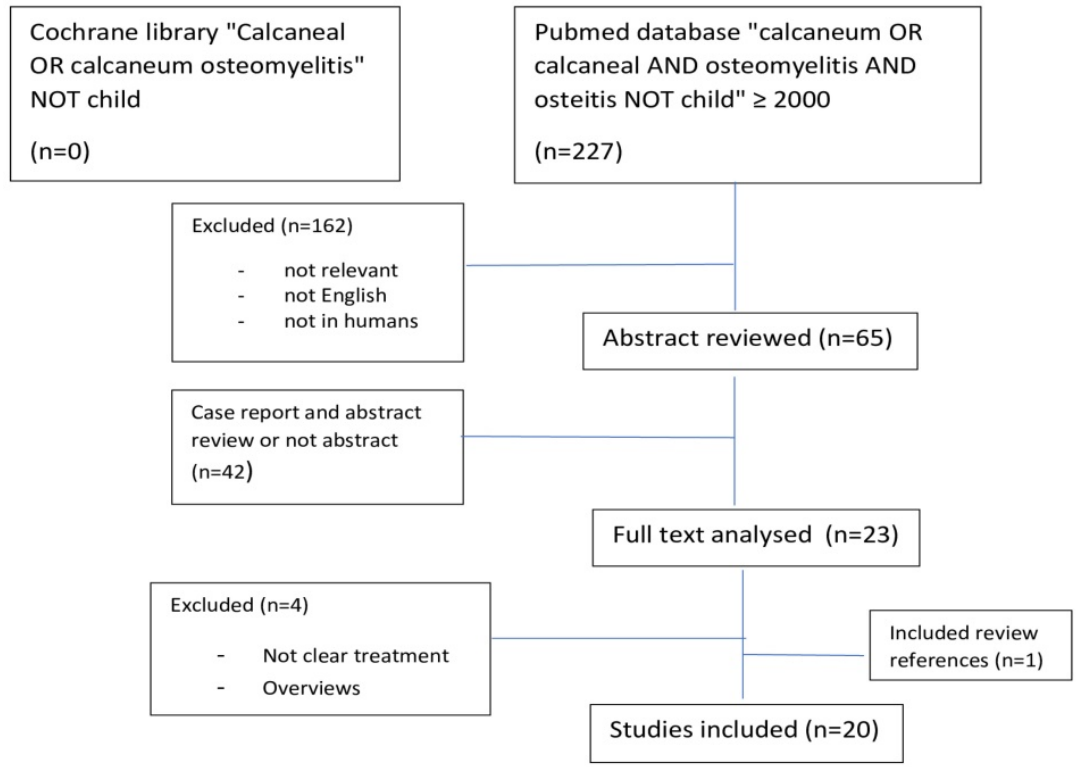

Figure 3. PRISMA flow diagram

\section{Results}

All the included studies gave a clear description of the surgical treatment received. Of the 20 studies, seven $(35 \%)$ described bone treatment only, six $(30 \%)$ focused on soft tissue treatment, five $(25 \%)$ on both soft tissue and bone treatment and two (10\%) described prognostic factors and differences in outcomes between diabetic and non-diabetic patients. All the studies were retrospective except for a prospective study by Paola et al., and a systemic review by Shade, consisting of 16 studies with 100 patients, most treated before 2000 . The retrospective studies had an average number of 15.3 patients [range 2 to 42]. Diagnosis of calcaneal osteomyelitis was made either by Magnetic Resonance Imaging (MRI) or confirmed by cultures.

\section{Bone treatment}

A total of 12 studies described the management of infected bone. Of these, five studies also reported the management of soft tissues. Of the seven studies focusing on bone treatment only, five used a specific technique: four studies assessed the outcome after partial calcanectomy and one after the Silo technique. A summary of these studies is shown in Table 1 and Table 2.

\section{Partial Calcanectomy}

In the study by Bollinger et al. (2002), the 22 included patients showed no recurrences of deep infection (9). However, more than $50 \%$ of the patients $(n=12)$ had delayed wound healing, and the poor wound healing rate was even higher $(100 \%)$ when considering diabetic patients only. Fisher et al. (2010) who assessed a novel approach using the 'Hurricane incision' in two patients showed no recurrence of infection but a $50 \%$ wound breakdown rate (10). This approach consists of a plantar based incision, with 2 semielliptical incisions circumscribing the calcaneal ulceration in order to resect the ulcer in total.

In the study by Oliver et al. (2015), 42 patients with calcaneal osteomyelitis and partial calcanectomy were included (11). The patients were divided into two cohorts with a resection of the calcaneum of $<50 \%$ and $>50 \%$. The lower extremity function and the limb salvage outcome were compared between both groups. They showed that regardless of the resection size, patients have similar function and a similar recurrence rate.

Brooks et al. (2004) reported 17 patients (20 feet) with calcaneal osteomyelitis treated either with partial or total calcanectomy (12). None of the patients had a recurrence of infection. Thirteen patients $(76 \%)$ were diabetic; nine of those $(69 \%)$ had surgery previously due to vasculopathy. The healing rate was $65 \%$, while the amputation rate was $30 \%$.

\section{Silo Technique}

The Silo technique consists of an MRI guided debridement, drilling bone tunnels and injecting antibiotic-loaded bioceramic (13). The study by Drampalos et al. (2017) includes 12 patients, in which either a primary wound closure was done, or a negative pressure wound therapy (NPWT) was used. All the wounds healed, except in one patient where a reverse sural flap was needed at a second stage. None of the patients developed an infection recurrence and only two $(17 \%)$ patients showed a decreased ambulatory status. 
Table 1: Systematic review of studies describing bone treatment for calcaneal osteomyelitis. Treatment

\begin{tabular}{|c|c|c|c|c|c|c|}
\hline Reference & Patients (no.) & Diabetes (no.) & Bone treatment & $\begin{array}{l}\text { Antibiotic } \\
\text { carrier }\end{array}$ & Soft tissue closure & Postoperative antibiotic \\
\hline $\begin{array}{l}\text { Bollinger et al. } \\
2002\end{array}$ & 22 & 9 & PC & None & Primary closure & NA \\
\hline Brooks et al. 2004 & 17 (20 feet) & 13 & $15 \mathrm{PC}$ and $5 \mathrm{TC}$ & None & Primary closure or NPWT & 6 weeks culture specific \\
\hline Fisher et al. 2010 & 2 & 2 & $\begin{array}{l}\text { PC with Hurricane } \\
\text { approach }\end{array}$ & None & Primary closure & NA \\
\hline Schade 2012 & 100 (16 papers) & NA & $76 \mathrm{PC}$ and $28 \mathrm{TC}$ & NA & NA & NA \\
\hline Oliver et al. 2015 & 42 & $\begin{array}{l}\text { Cohort 1: } 20 / 25 \\
\text { Cohort 2: } 15 / 17\end{array}$ & $\begin{array}{l}\text { Cohort 1: }<50 \% \text { PC } \\
\text { Cohort 2: }>50 \% \text { PC }\end{array}$ & none & Delayed primary closure & NA \\
\hline Babiak et al. 2016 & 32 & NA & $\begin{array}{l}\text { Bone preserving techniques } \\
\text { vs PC and TC }\end{array}$ & CGS & NA & $\begin{array}{l}2 \text { weeks empirical iv or oral }+4 \\
\text { weeks culture specific }\end{array}$ \\
\hline $\begin{array}{l}\text { Drampalos et al. } \\
2017\end{array}$ & 12 & 12 & Silo technique & $\begin{array}{l}\text { Gentamicin } \\
\text { loaded } \\
\text { bioceramic }\end{array}$ & Primary closure or NPWT & $\begin{array}{l}\text { Empiric gentamicin + teicoplanin } \\
\text { and modified accordingly for } 6 \text { to } \\
12 \text { weeks }\end{array}$ \\
\hline $\begin{array}{l}\text { Fraccalvieri et al. } \\
2012\end{array}$ & 7 & 2 & PC & None & $\begin{array}{l}\text { Integra dermal regeneration } \\
\text { template + NPWT for } 14 \text { days+ skin } \\
\text { graft }\end{array}$ & $\begin{array}{l}\text { After both procedures protocol } \\
\text { antibiotic, when cultures } \\
\text { available a specific antibiotic }\end{array}$ \\
\hline Gohds et al. 2012 & 3 & 0 & $\begin{array}{l}\text { Radical debridement with } \\
\text { cortical shell preservation }\end{array}$ & None & NPWT + gracilis free flap & Culture specific antibiotic \\
\hline Goudie et al. 2012 & 21 & 21 & PC & None & $\begin{array}{l}\text { NPWT + } \\
\text { 1) split thickness graft, 2) allogenic } \\
\text { bilayered skin subsitute or } \\
\text { 3) rhPDGF/ORC/coll. }\end{array}$ & NA \\
\hline Paola et al. 2016 & 18 & 1 & PC & None & $\begin{array}{l}\text { External frame + NPWT + dermal } \\
\text { substitute + split thickness skin } \\
\text { graft }\end{array}$ & $\begin{array}{l}2 \text { weeks iv followed by } 4 \text { weeks } \\
\text { oral }\end{array}$ \\
\hline Akkurt et al. 2017 & 23 & 23 & MRI guided debridement & None & External frame & NA \\
\hline
\end{tabular}

Table 2: Systematic review of studies describing bone treatment for calcaneal osteomyelitis. Results and follow up.

\begin{tabular}{|c|c|c|c|c|}
\hline Reference & Infection Recurrence & Amputation & Functional outcome & Follow up (months) \\
\hline Bollinger et al. 2002 & 0 & 0 & NA & $27(2-80)$ \\
\hline Brooks et al. 2004 & $35 \%$ & $6(30 \%)$ & NA & NA \\
\hline Fisher et al. 2010 & NA & 0 & NA & NA \\
\hline Schade 2012 & NA & NA & $85 \%$ maintain or increase ambulatory status & 33 \\
\hline Oliver et al. 2015 & NA & $\begin{array}{l}\text { Cohort 1: } 7(28 \%) \\
\text { Cohort 2: } 5(29 \%)\end{array}$ & $\begin{array}{l}\text { LEFS } \\
\text { Cohort 1: } 33.9 \\
\text { Cohort 2: } 36.6\end{array}$ & $\begin{array}{l}\text { Cohort 1: } 43 \\
\text { Cohort 2: } 38\end{array}$ \\
\hline Babiak et al. 2016 & $\begin{array}{l}\text { Bone preserving: } 23.5 \% \\
\text { PC or TC: } 11.8 \%\end{array}$ & $4(12.5 \%)$ & $\begin{array}{l}\text { Walking ability } \\
\text { Bone preserving: } 100 \% \\
\text { PC or TC: } 93.33 \%\end{array}$ & Minimal of 24 months \\
\hline $\begin{array}{l}\text { Drampalos et al. } \\
2017\end{array}$ & 0 & 0 & $6 / 8$ maintain ambulatory status & 4 \\
\hline $\begin{array}{l}\text { Fraccalvieri et al. } \\
2012\end{array}$ & 0 & 0 & All patients maintain preoperative mobility & $22(6-36)$ \\
\hline Gohds et al. 2012 & 0 & 0 & All patients return to ambulatory status & NA \\
\hline Goudie et al. 2012 & $24 \%$ & $4(24 \%)$ & $57 \%$ independent ambulation & 24 \\
\hline Paola et al. 2016 & 0 & 0 & NA & 7.5 \\
\hline Akkurt et al. 2017 & $5(22 \%)$ & $2(8.7 \%)$ & $78 \%$ were able to ambulate & 4.5 \\
\hline
\end{tabular}

\section{Combined Techniques}

Babiak et al. (2016) analysed 32 patients for calcaneal osteomyelitis. Of these, eight patients $(25 \%)$ were treated with a drilling operation combined with insertion of collagen-gentamicin sponges, nine $(28 \%)$ with debridement with or without fasciocutaneous flap, and $15(47 \%)$ with a partial calcanectomy. Infection control and wound healing was achieved in $75 \% \quad(n=6 / 8), 78 \% \quad(n=7 / 9)$, and $73 \% \quad(n=11 / 15)$, respectively (14). Patients treated with bone preservation showed lower infection control rates as well as lower patient satisfaction. However, they had a better walking ability. Failure was treated with total calcanectomy $(\mathrm{n}=2)$, amputation $(\mathrm{n}=4)$, or a further bone preserving technique $(\mathrm{n}=2)$.

In two studies $(15,16)$, Ilizarov circular frames were used to off-load and maintain the foot in equinus position to decrease the plantar soft tissue defect. In the study by Akurtt et al. (2017), they assessed the outcome of 23 patients treated with an Ilizarov frame. The external frame was removed after a mean of 8.2 
weeks, decreasing plantar flexion by $1^{\circ}$ per day. They reported pin site infections in $69.5 \%(n=16)$; in two a removal of the offending wires was necessary. Paola et al. (2016) included 18 patients with a mean external frame time of 11 weeks. The amount of correction per day is not mentioned, nor the pin site infection rate. Both studies agreed on the importance of bone debridement but differ in the technique. The first study used an MRI guided curettage with a $78 \%$ of clinical complete cure and $22 \%$ partial recovery ( 3 needed a flap operation and 2 a BKA). The second, advocated partial calcanectomy along with the application of a dermal substitute. They showed a $100 \%$ healing rate with no infection recurrence or need of amputation. However, three patients had a wound breakdown.

\section{Systematic Review}

The last of these seven studies is a systematic review by Schade (2012) including 16 articles (also case reports), mainly published before 2000 comparing the outcome of partial and total calcanectomy (17). The patients after total calcanectomy showed a higher complication rate, amputation rate, and need for ambulatory care in comparison to the patients with partial calcanectomy. Diabetic patients had a five times greater risk of amputation, but variation in the severity of the infection was not fully described.

The other three studies described the management of both bone and soft tissue. All of them were treated with partial calcanectomies, but different variants of soft tissue coverage. For this reason, the results will appear in the next section.

\section{Soft tissue}

A total of 11 studies discuss the soft tissue management for calcaneal osteomyelitis. Five studies reported both soft tissue and bone management (two of them have already been discussed in the bone treatment section), while six are more focused on soft tissue management. A summary of these studies is shown in Table 3 and Table 4.

Table 3: Systematic review of studies describing soft tissue treatment for calcaneal osteomyelitis. Treatment

\begin{tabular}{|c|c|c|c|c|}
\hline Reference & Infection Recurrence & Amputation & Functional outcome & Follow up (months) \\
\hline Al-Qattan 2001 & 0 & 0 & NA & 36 \\
\hline Yildirim et al. 2003 & $1(11 \%)$ & 0 & $8 / 9$ returned to presurgical ambulatory status & $22(15-27)$ \\
\hline Chen et al. 2005 & 0 & 0 & $100 \%$ returned to presurgical ambulatory status & $(6-12)$ \\
\hline Xu et al. 2009 & 0 & 0 & AOFAS score after 6 months: 76 & $34(6-72)$ \\
\hline Boffeli et al. 2013 & 0 & 0 & $100 \%$ returned to presurgical ambulatory status & NA \\
\hline Wang et al. 2014 & 0 & 0 & NA & $12(6-22)$ \\
\hline $\begin{array}{l}\text { Fraccalvieri et al. } \\
2012\end{array}$ & 0 & 0 & All patients maintain preoperative mobility & $22(6-36)$ \\
\hline Gohds et al. 2012 & 0 & 0 & All patients return to ambulatory status & NA \\
\hline Goudie et al. 2012 & $24 \%$ & $4(24 \%)$ & $57 \%$ independent ambulation & 24 \\
\hline Paola et al. 2016 & 0 & 0 & NA & 7.5 \\
\hline Akkurt et al. 2017 & $5(22 \%)$ & $2(8.7 \%)$ & $78 \%$ were able to ambulate & 4.5 \\
\hline
\end{tabular}

Table 4: Systematic review of studies describing soft tissue treatment for calcaneal osteomyelitis. Results and follow up.

\begin{tabular}{|c|c|c|c|c|c|c|}
\hline Reference & Patients (no.) & Diabetes (no.) & Bone treatment & Antibiotic carrier & Soft tissue closure & Postoperative antibiotic \\
\hline Al-Qattan 2001 & 4 & 0 & Debridement & None & Abductor digiti minimi & NA \\
\hline Yildirim et al. 2003 & 9 & 6 & $\begin{array}{l}\text { PC with cortical } \\
\text { shell preservation }\end{array}$ & None & Sural or saphenous neurocutaneous flap & $\begin{array}{l}3-4 \text { weeks iv followed by } 2-3 \text { weeks } \\
\text { oral treatment }\end{array}$ \\
\hline Chen et al. 2005 & 11 & 11 & Debridement & None & Sural fasciomusculocutaneous flap & Adjusted according culture results \\
\hline Xu et al. 2009 & 13 & 0 & $\begin{array}{l}\text { Removal of } \\
\text { sequestra and } \\
\text { resection od scarred } \\
\text { and infected bone }\end{array}$ & None & Free serratus muscle flap + skin graft & $\begin{array}{l}\text { Iv treatment based on culture } \\
\text { results }\end{array}$ \\
\hline Boffeli et al. 2013 & 3 & 0 & PC & None & Rotational flap closure & NA \\
\hline Wang et al. 2014 & 5 & 0 & Debridement & None & $\begin{array}{l}\text { Sural flap with adiponeurofascial } \\
\text { portion filling the bone cavity and } \\
\text { neurofasciocutaneous portion covering } \\
\text { the wound }\end{array}$ & $\begin{array}{l}4 \text { weeks iv }+2 \text { weeks oral treatment } \\
\text { adapted to cultures }\end{array}$ \\
\hline $\begin{array}{l}\text { Fraccalvieri et al. } \\
2012\end{array}$ & 7 & 2 & PC & None & $\begin{array}{l}\text { Integra dermal regeneration template }+ \\
\text { NPWT for } 14 \text { days }+ \text { skin graft }\end{array}$ & $\begin{array}{l}\text { After both procedures protocol } \\
\text { antibiotic, when cultures available a } \\
\text { specific antibiotic }\end{array}$ \\
\hline Gohds et al. 2012 & 3 & 0 & $\begin{array}{l}\text { Radical debridement } \\
\text { with cortical shell } \\
\text { preservation }\end{array}$ & None & NPWT + gracilis free flap & Culture specific antibiotic \\
\hline Goudie et al. 2012 & 21 & 21 & PC & None & $\begin{array}{l}\text { NPWT + } \\
\text { 1) split thickness graft, 2) allogenic } \\
\text { bilayered skin substitute or }\end{array}$ & NA \\
\hline
\end{tabular}




\begin{tabular}{llllll}
\hline Paola et al. 2016 & 18 & 1 & PC & None & $\begin{array}{l}\text { 3) rhPDGF/ORC/coll. } \\
\text { External frame + NPWT + dermal } \\
\text { substitute + split thickness skin graft } \\
\text { External frame }\end{array}$ \\
Akkurt et al. 2017 & 23 & 23 & $\begin{array}{l}\text { MRI guided } \\
\text { debridement }\end{array}$ & None & NA
\end{tabular}

*Note. NA: not available, PC: partial calcanectomy, NPWT: negative pressure wound therapy, rhPDGF/ORC/coll: recombinant platelet-derived growth factor/oxidised regenerated cellulose/bovine collagen, AOFAS: American Orthopaedic Foot \& Ankle Society

Below middle line studies describing both bone and soft tissue treatment

\section{Free Tissue Transfer}

Two studies $(18,19)$ assessed the outcome after radical bone debridement and soft tissue reconstruction using a free muscle flap, serratus anterior $(n=13)$ and gracilis muscle $(n=3)$, respectively. The degree or method of bone resection was not described. None of the cases had a recurrence of infection. In one patient, a haematoma occurred below the flap after surgery which was managed conservatively.

\section{Local Pedicled Flaps}

Al-Qattan (2001) used abductor digiti minimi muscle for soft tissue reconstruction in four patients with calcaneal osteomyelitis. Neither an infection recurrence nor postoperative changes of the neurological status were described (20). Bofelli et al. (2013) reported three cases treated by near total calcanectomy with different rotational flaps depending on the ulceration zone of the heel (21). No infection recurrence or complications were described, and all patients maintained their previous ambulatory status.

In three studies (22-24), patients were treated with aggressive bone and soft tissue debridement followed by reconstruction of the soft tissue defect using neurocutaneous or fasciomusculocutaneous flaps for a well vascularised environment. In all patients (Yildirim et al [ $\mathrm{n}=9$; neurocutaneous flap], Chen et al. $[n=11$; distally based sural fasciomusculocutaneous flap], Wang et al. [ $n=5$; "hybrid" sural flap]), a complete survival of the flaps was demonstrated. In the study by Yildirim et al. (2003) two patients with flap related complications due to venous congestion and partial loss at the donor site were described. Both were managed non-surgically. One patient $(11 \%)$ continued with persistent pyogenic discharge requiring a partial calcanectomy and a free muscle flap transfer. No study reported a recurrence of infection or amputation.

\section{Novel Techniques}

Goudie et al. (2012) published a study comparing three different techniques for soft tissue management after partial calcanectomy(25): split thickness graft $(n=5)$, allogenic bilayered skin substitute $(n=2)$ or recombinant platelet-derived growth factor/oxidised regenerated cellulose/bovine collagen (rhPDGF/ORC/collagen) $\quad(n=14)$. All patients in the first and second technique closure groups showed inadequate wound healing needing the secondary use of rhPDGF. Hence, the use of rhPDGF showed a higher primary healing rate $(97 \%)$. No recurrence of infection was described. Nonetheless, three patients needed BKA due to reopening of the ulcer within 12 months of healing. Overall, limb salvage rate was $76 \%$.

Two studies $(15,26)$ reported the use of dermal substitute layers without infection recurrence. However, $20 \%$ of the patients needed a skin graft or a second dermal layer implantation due to wound healing disorders. The last three studies used NPWT between bone debridement and rhPDGF or dermal layer application.

Negative pressure wound therapy (NPWT) was used in combination with other techniques in 6 studies $(12,13,15,19,25,26)$. Specific details (duration of use, sponge changes, complications) of NPWT were generally not reported, but Fraccalvieri et al.(2012) used it for 14 days prior to skin grafting. Overall, recurrence rates and amputation rates were not improved compared with those cases without NPWT use.

\section{Prognostic factors}

Two studies specifically investigated risk factors for poor outcome after treatment of calcaneal osteomyelitis. Walsh et al. (2013), showed that even with the same treatment, diabetic patients had a prolonged hospital stay and a lower limb salvage rate compared with non-diabetic patients (27). Merlet et al. (2014) described a 40.5\% relapse rate needing multiple and different treatments strategies. Additionally, 33\% of the patients presented unfavourable outcome with persistent heel ulceration or amputation (1). Risk factors for poor outcome after surgical treatment of calcaneal osteomyelitis were age $>65$, ASA Grade $>2$, diabetes, neuropathy and posttraumatic aetiology.

\section{Surgical stages}

Nine studies described treatment in a single stage $(9,10,13,14,16,18,21,23,24)$. Only 2 papers describe 2 stage procedures $(11,20)$. In both papers, skin closure was performed separately after the first stage bone excision. Finally, 4 papers report a 
multiple stage technique ( $\geq 3$ stages) $(15,19,25,26)$.

\section{Antibiotic}

\section{Local antibiotic delivery}

In the study by Babiak et al. (2016), eight (25\%) of 32 patients were treated with collagen-gentamicin sponges (CGS) as a local antimicrobial carrier after debridement and drilling of calcaneum (14). Two $(25 \%)$ of these patients had a recurrence of infection and were finally treated with BKA, neither being able to walk after amputation. The remaining six (75\%) patients had no further surgical treatment for infection recurrence and were able to walk and satisfied with their management.

Drampalos et al. (2017) used in addition to the above mentioned Silo technique an antibiotic carrier consisting of calcium sulphate (CS) and hydroxyapatite (HA) loaded with gentamicin (13). None of the patients showed infection recurrence.

Lastly, Walsh et al. (2013), also used two different localized delivery systems (CS with tobramycin sulphate or gentamicin-collagen haemostat) in all 10 patients included (27). The choice of one system over the other depended on the nature of the wound, previous wound isolates and blood cultures. In their study, diabetic patients performed worse than non-diabetics. Two patients required a BKA after multiple debridements due to infection persistence. Infection recurrence was not properly stated.

\section{Antimicrobial therapy after surgery}

Twelve studies reported the use of systemic antimicrobial therapy after surgery. All used antibiotics selected according to culture results and antibiogram. However, duration and empiric treatment are not always mentioned. Only nine studies described the specific duration ranging from two to 12 weeks. In three studies $(1,22,27)$, the duration was based on cultures results, wound and soft tissue progression, and blood markers. Limited specific data on microbiology was reported in only four studies $(14,15,16,23)$. Bone cultures demonstrated Staphylococcus aureus to be the most common organism involved in calcaneal osteomyelitis.

\section{Discussion}

According to the evidence reviewed over the last 18 years, calcaneal osteomyelitis treatment tried to deliver the following objectives: (1) bone infection control (surgical excision and systemic antimicrobial treatment), (2) dead space and wound management (with or without local antibiotic delivery and wound closure) and (3) a good functional outcome.

Limitations of the included studies are first, the lack of standardized definitions of infection, failure, relapse, recurrence of infection, and wound healing disorders. In some studies, detailed information about important diagnostic protocols, outcome parameters, such as recurrence of infection or functional results, is completely absent. Many studies report no recurrence of infection but list numerous patients with "poor wound healing" or "wound breakdown". Clearly some of these will have either persistence of the initial infection or recurrence as the cause of the open wound. There is a need for a standardized method of reporting wound problems and careful use of a definition for recurrent infection. Moreover, there is an important potential bias because the extent of the infection is not fully described in all the papers and therefore, may be variable.

Secondly, most of the studies were analysed retrospectively $(n=19 / 20)$, only one study was conducted prospectively. Another drawback is the small sample size of assessed patients with calcaneal osteomyelitis and the short follow-up. Ideally, all patients should be reviewed for at least one year, but this was only reported in 10 studies. The number of investigated patients ranged from 2 to $100,80 \%$ of the studies included less than 30 patients and 35\% less than 10 patients. Due to these limitations, a proper comparison between these studies is difficult and an accurate and reasonable conclusion cannot always be guaranteed. There may be a significant bias in favour of good outcomes with smaller studies reporting no complications.

In this systematic review, 20 studies matching our inclusion criteria were studied in detail. Different surgical approaches related to bone and/or soft tissue management achieved similar reinfection rates and functional outcome. This review has not identified any method which is superior to any other, although partial bone resections may offer better functional outcome compared to total calcanectomy. A study by Brown et al. (28) on diabetic patients with foot ulcers who underwent various levels of amputation such as transtibial, transmetatarsal, Chopart, partial calcanectomy (PC) total calcanectomy (TC) has shown no significant differences in ambulatory status though numbers were small. Interestingly, mortality rates at 5 years for TTA was $45 \%$, for PC was $69 \%$, for TC was $59 \%$ and more proximal amputations were required in $5 \%$ for TTA $35 \%$ for PC and $31 \%$ for TCs, demonstrating the poor outcomes whatever the procedure in the diabetic population (28).

Regarding bone management results, bone preserving and more radical procedures seem to have comparable relapse and recurrence rates of osteomyelitis, although functional results may be better with less radical surgeries $(11,13,14)$. However, 
the functional outcome was not always specified. Only the study by Oliver et al. (2015) assessed the lower limb function objectively by using the Lower Extremity Function Scale (LEFS), in which patients with less aggressive surgeries provided better LEFS functional scores. Other studies assessed the walking capability of their patients and compared it with their preoperative walking ability $(9,13,14,17-23,25,26)$. These studies generally show preservation or improvement of the functional outcome after surgery.

Wound closure can be achieved by various plastic procedures if a primary wound closure is not possible: free muscle flaps (serratus anterior, gracilis), or local flaps (rotational flaps, abductor digiti minimi flap, neurocutaneous or fasciomusculocutaneous flaps). The reviewed studies showed no difference in the reinfection rate and failure rate of the flaps. However, the choice of soft tissue coverage should be based on the location and size of the soft tissue defect (21). Direct closure with the adjacent normal specialized skin is preferable, but small defects may be reliably covered by local pedicle flaps.

No recommendation on the extent of soft tissue defect which can be covered by a local flap can be given from the reviewed literature. In larger defects, free flaps which provide a good obliteration of dead space should be used. They also import well perfused tissue which can deliver antibiotics and immune cells to the area. Disadvantages of free flaps are the need of microsurgery, long operation time, and prolonged hospital stay combined with higher costs. They are also usually insensate, producing a later risk of pressure ulceration. Regardless of which coverage is used, the applied procedure should guarantee an improved bone vascularization and a good dead space management to avoid haematoma formation (8).

NPWT showed no additional benefit in the treatment of calcaneal osteomyelitis. This finding is similar to the experience of NPWT in open fractures (29). A drawback of NPWT is the need for at least one additional surgery for wound closure and often the sponge will need several exchanges, prior to definitive closure $(13,15,19,25,26)$. Furthermore, prolonged use of NPWT can lead to an increased surgical site infection (SSI) rate due to sponge seeding. However, the current literature on this field is controversial and the development of SSI after NPWT depends on wound type and duration of use $(29,30$, 31).

The use of external circular frames has shown good results. It seems that this device allows off-loading of the hindfoot and maintains the foot in an equinus position to minimize the soft tissue defect $(15,16)$. However, a standardized treatment algorithm is missing.

The novel use of rhPDGF is an interesting approach which may be helpful in more recalcitrant cases (25).

Only two-thirds of the studies provide a proper description of the antimicrobial therapy used. Generally, an empirical antimicrobial course was given initially after surgery followed by an oral regime based on the microbiological results and antibiogram. For successful infection control, a combined surgical and antimicrobial treatment can be highly recommended and has been well-described in the literature in a wide range of types of bone infection $(2,32,33)$. The use of local antibiotic carriers for osteomyelitis shows promising results in the current literature (33). If antibiotic carriers were used in the calcaneum, less radical surgeries were necessary, the osteomyelitis was controlled, and the patients were highly satisfied $(13,14,27)$. However, the numbers reported are small and this modality of antibiotic delivery requires further study.

Calcaneal osteomyelitis can be associated with various comorbidities. Neuropathy and vasculopathy are known to reduce soft tissue healing resulting in higher recurrence and amputation rates $(1,27)$. Merlet et al. (2014), found several prognostic factors for a poorer outcome including neuropathy and diabetes, age $>65$ years, ASA $>2$ and posttraumatic origin of the infection. Interestingly, vasculopathy was not reported to be a risk factor for wound healing disorders alone, although diabetes (which often produces vascular insufficiency) was included.

Healing rates after treatment of calcaneal osteomyelitis range from $66 \%$ to $100 \%$ in all included studies, although every article provided different definitions. Some considered that healing was achieved if there was no need for multiple debridements; others preferred using clinical and/or radiological signs of ongoing infection even if the patient needed repeated debridements or different surgical approaches to control the infection. Studies which investigated diabetic patients generally showed a reduced healing rate.

Overall results favour less radical surgery, with good soft tissue coverage and dead space management. Late recurrences remain challenging, especially in patients with continued neuropathy and new ulceration.

Calcaneal osteomyelitis is difficult to manage and requires a multidisciplinary approach involving orthopaedic surgeons, plastic surgeons and infectious diseases physicians. Studies with standardized treatment algorithms and outcome measures including inputs from all three disciplines would be valuable in clinical practice. However, patients with 
this condition have varied co-morbidities and different components of disease in the calcaneum, requiring an individual treatment strategy.

\section{Acknowledgements}

All authors contributed to study design and draft and/or revision of the manuscript.

\section{Competing Interests}

The authors have declared that no competing interest exists.

\section{References}

1. Merlet A, Cazanave C, Dauchy F, Dutronc HÉ, Casoli V, Chauveaux D, Barbeyrac B., Dupon M. Prognostic factors of calcaneal osteomyelitis. Scand J Infect Dis. 2014;46:555-560.

2. Sanders J, Mauffrey C. In trauma update Long Bone Osteomyelitis in Adults: Fundamental Concepts and Current Techniques. Orthopedics. 2013 May;36(5):368-75.

3. Gaenslen FJ. Split-heel approach in osteomyelitis of os calcis. JBJS[Am]. 1931;13:759-65

4. Pinzur MS, Gold J, Schwartz D, Gross N. Energy demands for walking in dysvascular amputees as related to the level of amputation. Orthopedics. 1992;15(9):1033-1037.

5. Desveaux L, Goldstein RS, Mathur S, Hassan A, Devlin M, Pauley T, et al. Physical activity in adults with diabetes following prosthetic rehabilitation. Can J Diabetes. 2016;40(4):336-341.

6. Aulivola, B; Hile, CN; Hamdan, AD; et al. Major lower extremity amputation: outcome of a modern series. Arch Surg. 2004;139:395-399.

7. Fukuda T., Reddy V., Ptaszek AJ. The infected calcaneus. Foot Ankle Clin. 2010 Sep;15(3):477-86.

8. Attinger C., Cooper P. Soft tissue reconstruction for calcaneal fractures or osteomyelitis. Orthop Clin North Am. 2001;32(1):135-170.

9. Bollinger M., Thordarson D. B. Partial calcanectomy: an alternative to below knee amputation. Foot Ankle Int. 2002 Oct;23(10):927-32.

10. Fisher TK, Armstrong DG. Partial calcanectomy in high-risk patients with diabetes: use and utility of a "hurricane" incisional approach. Eplasty. 2010;140-51.

11. Oliver NG, Steinberg JS, Powers K, Evans KK, Kim PJ, Attinger CE. Lower extremity function following partial calcanectomy in high-risk limb salvage patients. J Diabetes Res. 2015;2015(4):1-7.

12. Brooks KR, Lin SS, Berberian WS, Abidi N a, Vieira P. Calcanectomy for Treatment of the Infected Os Calcis. Tech Foot Ankle Surg. 2004;3(3):165-76.

13. Drampalos E, Mohammad HR, Kosmidis C, Balal M, Wong J, Pillai A. The Foot Single stage treatment of diabetic calcaneal osteomyelitis with an absorbable gentamicin-loaded calcium sulphate / hydroxyapatite biocomposite: The Silo technique. Foot. 2018;34:40-4.

14. Babiak I, Pedzisz P, Kulig M, Janowicz J, Maldyk P. Comparison of Bone Preserving and Radical Surgical Treatment in 32 Cases of Calcaneal Osteomyelitis. J Bone Jt Infect. 2016; 1:10-16.

15. Paola LD, Carone A, Boscarino G, Scavone G, Vasilache L. Combination of Open Subtotal Calcanectomy and Stabilization With External Fixation as Limb Salvage Procedure in Hindfoot-Infected Diabetic Foot Ulcers. Int J Low Extrem Wounds. 2016 Dec;15(4):332-337

16. Akkurt MO, Demirkale I, Öznur A. Partial calcanectomy and Ilizarov external fixation may reduce amputation need in severe diabetic calcaneal ulcers. Diabet Foot Ankle. 2017;8(1):1264699.

17. Schade V.L. Partial or total calcanectomy as an alternative to below-the-knee amputation for limb salvage: a systematic review. J Am Podiatr Med Assoc. 2012;102(5):396-405.

18. $\mathrm{Xu} \mathrm{X,} \mathrm{Zhu} \mathrm{Y,} \mathrm{Liu} \mathrm{J.} \mathrm{Treatment} \mathrm{of} \mathrm{Calcaneal} \mathrm{Osteomyelitis} \mathrm{With} \mathrm{Free}$ Serratus Anterior Muscle Flap. Foot Ankle Int. 2009 Nov;30(11):1088-93.

19. Ghods M, Grabs R, Kersten C, et al. A Modified Free Muscle Transfer Technique to Effectively Treat Chronic and Persistent Calcaneal Osteomyelitis. Ann Plast Surg. 2012;68(6):599-605.

20. Al-Qattan M. Harvesting the Abductor Digiti Minimi as a Muscle Plug With the Lateral Calcaneal Artery Skin Flap. Ann Plast Surg. 2001 Jun;46(6):651-3.

21. Boffeli T. J., Collier R. C. Near total calcanectomy with rotational flap closure of large decubitus heel ulcerations complicated by calcaneal osteomyelitis. J Foot Ankle Surg. 2013;52(1):107-112
22. Yildirim S., Gideroğlu K., Aköz T. The Simple and Effective Choice for Treatment of Chronic Calcaneal Osteomyelitis: Neurocutaneous Flaps. Plast Reconstr Surg. 2003 Feb;111(2):753-60

23. Chen SL, Chen TM, Chou TD, Chang SC, Wang HJ. Distally based sural fascio-musculocutaneous flap for chronic calcaneal osteomyelitis in diabetic patients. Ann Plast Surg. 2005;54:44-8.

24. Wang C, Sun L. A " Hybrid" Sural Flap for Treatment of Chronic Calcaneal Osteomyelitis. J Reconstr Microsurg. 2014 Sep;30(7):457-62.

25. Goudie EB., Gendics C., Lantis JC 2nd. Multimodal therapy as an algorithm to limb salvage in diabetic patients with large heel ulcers. Int Wound J. 2012 Apr;9(2):132-8.

26. Fraccalvieri M, Pristera G, Zingarelli E, Ruka E, Bruschi S. Treatment of chronic heel osteomyelitis in vasculopathic patients. Can the combined use of Integra, skin graft and negative pressure wound therapy be considered a valid therapeutic approach after partial tangential calcanectomy?. Int Wound J. 2012;9:214-20.

27. Walsh TP, Yates BJ. The Foot Calcanectomy: Avoiding major amputation in the presence of calcaneal osteomyelitis - A case series. Foot (Edinb). 2013 Dec;23(4):130-5.

28. Brown ML, Tang W, Patel A, Baumhauer JF. Partial foot amputations in patients with diabetic foot ulcers. Foot Ankle Int. 2012 Sep;33(9):707-716.

29. Costa ML, Achten J, Bruce J, Tutton E, Petrou S, Lamb SE, et al. Effect of Negative Pressure Wound Therapy vs Standard Wound Management on 12-Month Disability Among Adults With Severe Open Fracture of the Lower Limb: The WOLLF Randomized Clinical Trial. JAMA. 2018;319(22):2280-2288

30. Tansarli GS, Vardakas KZ, Stratoulias C, Peppas G, Kapaskelis A, Falagas ME. Vacuum-Assisted Closure versus Closure without Vacuum Assistance for Preventing Surgical Site Infections and Infections of Chronic Wounds: A Meta-Analysis of Randomized Controlled Trials. Surg Infect (Larchmt). 2014;15(4):363-367.

31. Sendi $P$, McNally MA. Wound Irrigation in Initial Management of Open Fractures. N Engl J Med. 2016; 374(18): 1788-90.

32. McNally MA, Nagarajah K. Osteomyelitis Orthop Trauma. 2010; 24(6): 416-429

33. McNally MA, Ferguson JY, Lau ACK, Diefenbeck M, Scarborough M, Ramsden AJ, et al. Single-stage treatment of chronic osteomyelitis with a new absorbable, gentamicin-loaded, calcium sulphate/hydroxyapatite biocomposite: A prospective series of 100 cases. Bone Joint J. 2016 Sep;98B(9):1289-96. 